\title{
Insatisfação com a imagem corporal e relação com estado nutricional, adiposidade corporal e sintomas de anorexia e bulimia em adolescentes
}

\author{
Body image dissatisfaction and its relationship with nutritional status, body fat, and \\ anorexia and bulimia symptoms in adolescents
}

\section{Cilene Rebolho Martins ${ }^{1}$, Andreia Pelegrini², Silvana Corrêa Matheus ${ }^{3}$, Edio Luiz Petroski ${ }^{4}$}

${ }^{1}$ Mestranda, Programa de Pós-Graduação em Educação Física, Universidade Federal de Santa Catarina (UFSC), Florianópolis, SC. ${ }^{2}$ Doutoranda, Programa de PósGraduação em Educação Física, UFSC. ${ }^{3}$ Doutora. Professora adjunta, Centro de Educação Física e Desportos, Universidade Federal de Santa Maria (UFSM), Santa Maria, RS. ${ }^{4}$ Pós-Doutor. Professor titular, Centro de Desportos, UFSC.

\section{Resumo}

Objetivos: Identificar a prevalência de insatisfação com a imagem corporal e de sintomas de anorexia e bulimia em adolescentes do sexo feminino da cidade de Santa Maria (RS) e verificar a associação da insatisfação com a imagem corporal, estado nutricional, adiposidade corporal e sintomas de anorexia e bulimia.

Método: Foram analisadas 258 adolescentes do sexo feminino (11 a 13 anos), estudantes de escolas públicas. Aplicou-se o Body Shape Questionnaire e o Teste de Atitudes Alimentares (EAT-26). Foram calculados o índice de massa corporal e o percentual de gordura. Foram utilizados o teste quiquadrado e a regressão logística.

Resultados: A prevalência de insatisfação com a imagem corporal foi de $25,3 \%$ e a de sintomas de anorexia e bulimia foi de $27,6 \%$. A insatisfação com a imagem corporal apresentou associação com o estado nutricional, sendo que as adolescentes com excesso de peso apresentaram maior insatisfação (razão de chances = 2,64; intervalo de confiança de 95\% 1,02-6,83).

Conclusões: As prevalências de insatisfação corporal e sintomas de anorexia e bulimia foram elevadas. O estado nutricional parece ser o melhor preditor da insatisfação corporal.

Descritores: Imagem corporal, anorexia, bulimia, antropometria.

\begin{abstract}
Objectives: To identify the prevalence of body image dissatisfaction and anorexia and bulimia symptoms in adolescent girls from the city of Santa Maria, Brazil, and to determine the association of body image dissatisfaction with nutritional status, body fat, and anorexia and bulimia symptoms. Method: We investigated 258 adolescent girls (11 to 13 years) from public schools. The Body Shape Questionnaire and the Eating Attitudes Test (EAT-26) were used. Body mass index and percent body fat were calculated. The chi-square test and logistic regression were used.

Results: The prevalence of body image dissatisfaction was $25.3 \%$ and the prevalence of anorexia and bulimia symptoms was $27.6 \%$. Body image dissatisfaction was associated with nutritional status, with overweight girls presenting higher dissatisfaction $(\mathrm{OR}=2.64 ; 95 \% \mathrm{CI} 1.02-6.83)$.

Conclusions: The prevalence rates of body image dissatisfaction and anorexia and bulimia symptoms were high. Nutritional status seems to be a better predictor of body image dissatisfaction.
\end{abstract}

Keywords: Body image, anorexia, bulimia, anthropometry.

Correspondência:

Cilene Rebolho Martins, Rua Jornalista Tito Carvalho, 101/C2/203, Bairro Trindade, CEP 88040-480, Florianópolis, SC. Tel.: (48) 3721.8562, (48) 9133.3281. E-mail: cilenerebolho@yahoo.com.br

Não foram declarados conflitos de interesse associados à publicação deste artigo.

Copyright (C) Revista de Psiquiatria do Rio Grande do Sul - APRS

Recebido em 14/04/2009. Aceito em 16/09/2009. 


\section{Introdução}

A imagem corporal pode ser definida como a figura que se tem do próprio corpo e os sentimentos em relação ao seu tamanho, forma e partes constituintes ${ }^{1}$.

Com o surgimento da puberdade ocorre um maior acúmulo de gordura corporal no sexo feminino, o que, muitas vezes, pode desencadear uma maior insatisfação com a imagem corporal $^{2,3}$. Além disso, a mídia, os amigos, os pais e a sociedade, em geral, também influenciam nesse contexto, uma vez que impõem um padrão de beleza essencialmente magro ${ }^{4,5}$.

A insatisfação com a imagem corporal tem despertado grande interesse entre os pesquisadores, principalmente devido à sua estreita relação com os distúrbios alimentares tais como a anorexia e a bulimia.

Evidências têm demonstrado aumento na prevalência de insatisfação com a imagem corporal em adolescentes ${ }^{2,3,6}$. Da mesma forma, o número de casos de anorexia e bulimia nessa fase também tem aumentado ${ }^{2,3,5}$. Esses distúrbios alimentares são caracterizados pelo medo mórbido de engordar, pela preocupação obsessiva com os alimentos, pelo desejo persistente de emagrecer e pela distorção da imagem corporal $^{5,7,8}$, causando prejuízos biológicos, psicológicos e aumento da morbimortalidade .

As estimativas da prevalência de distúrbios alimentares variam de acordo com a metodologia e a amostra utilizada. De uma forma geral, a prevalência de anorexia varia entre 0,5 e $3,7 \%$ e a de bulimia, entre 1,1 e $4,2 \%{ }^{10}$. Geralmente, o perfil dos pacientes que desenvolvem esses distúrbios é de adolescente do sexo feminino, raça branca e alto nível socioeconômico, porém se tem observado que esse grupo é cada vez mais heterogêneo, tendo se realizado diagnósticos em pré-adolescentes e em pacientes de níveis econômicos mais baixos ${ }^{9}$.

Poucos estudos têm investigado a relação entre insatisfação com a imagem corporal e sintomas de distúrbios alimentares em adolescentes. Um estudo recente encontrou forte associação entre essas variáveis em escolares do sexo feminino na faixa etária de 10 a $19 \operatorname{anos}^{6}$, mas existe uma carência de pesquisas que sustentem esses resultados.

No que se refere ao estado nutricional, alguns estudos têm mostrado existir associação com a insatisfação corporal em adolescentes ${ }^{11}$ e em estudantes universitárias ${ }^{12}$. Entretanto, ainda há necessidade de investigar essa associação de forma mais detalhada, pois os estudos utilizaram ferramentas estatísticas bastante simplificadas (qui-quadrado). Quanto à adiposidade corporal, ainda não foi abordada na literatura a sua associação com a insatisfação com a imagem corporal em adolescentes.

Assim, os objetivos do presente estudo foram identificar a prevalência de insatisfação com a imagem corporal e de sintomas de anorexia e bulimia em adolescentes do sexo feminino da cidade de Santa Maria (RS) e verificar a associação da insatisfação com a imagem corporal com estado nutricional, adiposidade corporal e com a presença de sintomas de anorexia e bulimia.

\section{Método}

Trata-se de um estudo transversal, descritivo e de associação realizado em 2007 com adolescentes do sexo feminino de 11 a 13 anos, estudantes do ensino fundamental (sexta a oitava séries) de escolas públicas da cidade de Santa Maria (RS).

De acordo com o censo escolar realizado pelo setor de estudos e pesquisas educacionais do Ministério da Educação ${ }^{13}$, a população do estudo foi composta por 5.067 adolescentes. A amostra foi do tipo probabilística, estratificada por região geográfica (dividindo-se a cidade de Santa Maria em três regiões), rede de ensino (estadual e municipal) e idade (11, 12 e 13 anos).

Para o cálculo amostral, utilizou-se a equação de Fonseca \& Martins ${ }^{14}$, considerando-se erro relativo tolerável de $5 \%$ e intervalo de confiança de 95\% (IC95\%), obtendo-se um tamanho amostral estimado de 166 adolescentes. As escolas foram selecionadas aleatoriamente por sorteio simples, totalizando 15 instituições de ensino, sendo seis estaduais e nove municipais. A coleta de dados foi realizada por conglomerado de turmas, sendo convidadas a participar do estudo todas as adolescentes na faixa etária alvo presentes em sala de aula no dia da coleta. Por esse motivo, a amostra final do presente estudo foi composta por 258 adolescentes.

A coleta de dados foi realizada no ambiente escolar durante o período de aula das adolescentes, sendo dividida em duas etapas: 1) aplicação dos questionários de imagem corporal e atitudes alimentares; e 2) mensuração das medidas antropométricas.

A insatisfação com a imagem corporal foi avaliada por meio do Body Shape Questionnaire (BSQ) em sua versão para o português de Cordás \& Castilho ${ }^{15}$ do original elaborado por Cooper et al. ${ }^{16}$ e validada por Manetta ${ }^{17}$. Trata-se de um questionário autoaplicável do tipo escala de Likert, composto por 34 perguntas com seis opções de resposta, que pontuam de 1 a 6 , sendo a maior pontuação conferida àquelas respostas que refletem maior preocupação com a imagem corporal e maior autodepreciação devido à aparência física, especialmente no sentido de sentir-se com excesso de peso. A partir da pontuação obtida, as adolescentes foram classificadas em satisfeitas (escore $<111$ ) ou insatisfeitas (escore $\geq$ 111) (Cordás \& Castilho ${ }^{15}$ adaptado por Alves et al. ${ }^{6}$ ).

Para a avaliação dos sintomas de anorexia e bulimia, utilizou-se o Teste de Atitudes Alimentares (Eating Attitudes Test, EAT-26), proposto por Garner et al. ${ }^{18}$, traduzido para o português por Nunes et al. ${ }^{8}$ e validado por Bighetti ${ }^{19}$. O EAT-26 é um questionário de autorrelato, que possui questões do tipo escala de Likert com seis opções de resposta (nunca, quase nunca, poucas vezes, às vezes, muitas vezes e sempre), que pontuam de 0 a 3 . As respostas nunca, quase nunca e poucas vezes somam 0 pontos, e as respostas às vezes, muitas vezes e sempre pontuam 1, 2 e 3 pontos, respectivamente. Na questão 25 , a pontuação é conferida de forma inversa às demais, somando-se 0 pontos para as alternativas às vezes, muitas vezes e sempre, e 1, 2 e 3 pontos para as alternativas poucas vezes, quase nunca e nunca, respectivamente. A pontuação final do questionário pode variar de 0 a 78 pontos. No presente estudo, 
as adolescentes que somaram 20 pontos ou mais no EAT-26 foram classificadas com comportamento alimentar de risco, conforme proposto em outros estudos ${ }^{3,20,21}$. Dessa forma, o resultado do EAT-26 foi categorizado em: EAT positivo (EAT + ) para presença de sintomas de anorexia e bulimia e EAT negativo (EAT-) para ausência de sintomas de anorexia e bulimia.

$\mathrm{O}$ estado nutricional foi avaliado pelo índice de massa corporal (IMC) (massa corporal/estatura ${ }^{2}$ ). Para a mensuração da massa corporal e estatura, foram utilizados procedimentos padronizados $^{22}$. Os valores de IMC foram classificados utilizando-se pontos de corte amplamente aceitos na literatura: baixo peso (IMC $\left.<18,5 \mathrm{~kg} / \mathrm{m}^{2}\right)^{23}$, peso normal (IMC entre 18,5 e $24,9 \mathrm{~kg} / \mathrm{m}^{2}$ ) e excesso de peso (IMC $\left.\geq 25 \mathrm{~kg} / \mathrm{m}^{2}\right)^{24}$.

A adiposidade corporal foi determinada pelo percentual de gordura $(\% \mathrm{G})$, obtido com a mensuração das dobras cutâneas tricipital e subescapular ${ }^{25}$. Para o cálculo do $\% \mathrm{G}$, foi utilizada a equação de Slaughter et al $^{26}$. Essa variável foi classificada adaptando-se os pontos de corte estabelecidos por Lohman ${ }^{27}$ : normal (até $25 \%$ ); alto (de 26 a $35 \%$ ) e muito alto ( $>35 \%$ ).

A análise descritiva dos dados foi realizada por meio de média, desvio padrão e distribuição de frequências. Utilizouse o teste qui-quadrado para verificar a associação entre a insatisfação com a imagem corporal e as seguintes variáveis: estado nutricional, adiposidade corporal e sintomas de anorexia e bulimia. Essas associações também foram testadas, utilizando-se a análise de regressão logística com o cálculo da razão de chances (odds ratio, OR). A insatisfação com a imagem corporal foi o desfecho ou variável dependente do estudo. Foram testados dois modelos, um simples e um múltiplo (ajustado por todas as variáveis). A análise estatística foi realizada no programa SPSS 13.0, com nível de significância de 5\%.

Este estudo foi realizado com a autorização das Secretarias Municipal e Estadual de Educação da cidade de Santa Maria, sendo o projeto aprovado pelo Comitê de Ética em Pesquisa com Seres Humanos da Universidade Federal de Santa Maria (UFSM) ( $\mathrm{n}^{\mathrm{o}}$ de processo 23081.013844/200748), Santa Maria (RS). Para participarem do estudo, as adolescentes entregaram um termo de consentimento livre e esclarecido devidamente assinado pelo responsável.

\section{Resultados}

As características da amostra encontram-se na Tabela 1, na qual estão apresentados os valores de média, desvio padrão, valor mínimo e máximo.

Tabela 1 - Caracterização das variáveis idade, massa corporal, estatura, índice de massa corporal, percentual de gordura, BSQ e EAT-26 referentes às adolescentes estudantes de escolas municipais e estaduais da cidade de Santa Maria (RS)

\begin{tabular}{|c|c|c|c|}
\hline Variáveis & Média & Mínimo & Máximo \\
\hline Idade (anos) & $12,06 \pm 0,87$ & 11 & 13 \\
\hline Massa corporal (kg) & $47,39 \pm 10,4$ & 24,6 & 101,3 \\
\hline Estatura (m) & $1,55 \pm 0,08$ & 1,27 & 1,70 \\
\hline $\operatorname{IMC}(\mathrm{kg} / \mathrm{m} 2)$ & $19,64 \pm 3,5$ & 13,60 & 36,76 \\
\hline$\% \mathrm{G}(\%)$ & $28,73 \pm 9,79$ & 8,63 & 63,48 \\
\hline Pontuação do BSQ & $87,97 \pm 35,95$ & 34 & 191 \\
\hline Pontuação do EAT-26 & $15,37 \pm 12,9$ & 0 & 74 \\
\hline
\end{tabular}

$\% \mathrm{G}=$ percentual de gordura; BSQ = Body Shape Questionnaire; EAT-26 = teste de atitudes alimentares; IMC = índice de massa corporal

As prevalências de insatisfação com a imagem corporal e de sintomas de anorexia e bulimia foram de 25,3 e $27,6 \%$, respectivamente. Em relação ao estado nutricional, verificou-se que $68,8,8,2$ e $23 \%$ das adolescentes foram classificadas com peso normal, baixo peso e excesso de peso, respectivamente. Quanto ao \%G, 42,4\% das estudantes foram classificadas na categoria "normal", 34,6\% na “alto" e 23\% na "muito alto" (dados não apresentados).
A insatisfação com a imagem corporal apresentou associação com o estado nutricional (IMC) $(p<0,01)$ e com a adiposidade corporal $(\% \mathrm{G})(\mathrm{p}<0,01)$, verificados por meio do teste qui-quadrado (Figura 1). Observa-se que à medida que as classificações do IMC e \%G aumentam, ocorre um incremento na insatisfação com a imagem corporal nas adolescentes.

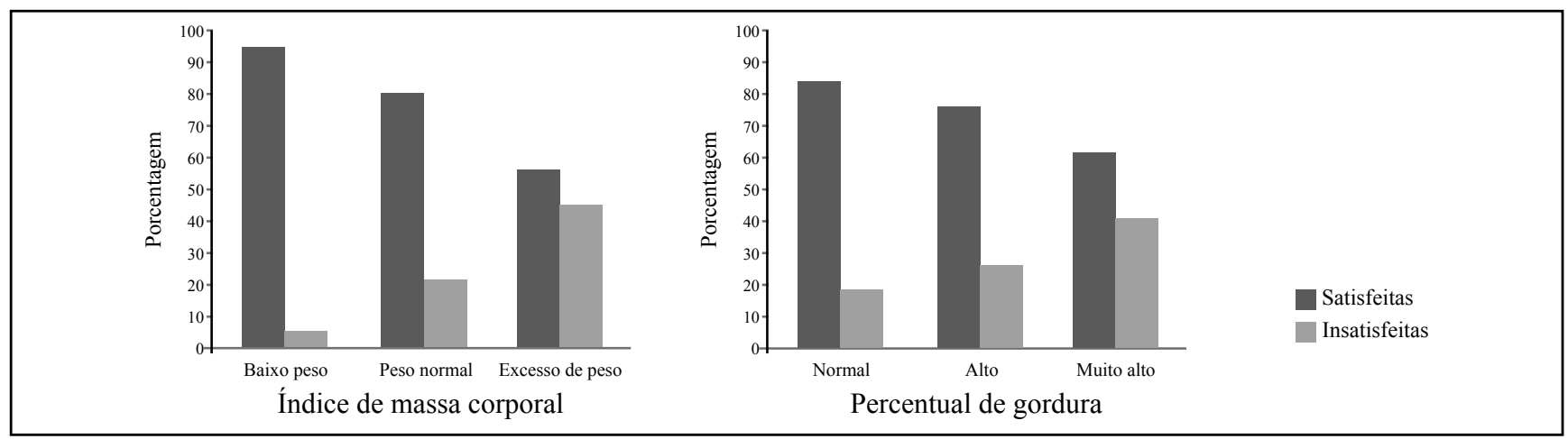

Figura 1 - Distribuição da insatisfação com a imagem corporal segundo os indicadores antropométricos (índice de massa corporal e percentual de gordura) de adolescentes do sexo feminino - Santa Maria (RS), 2007 
A Tabela 2 apresenta os resultados da análise de regressão logística para a insatisfação com a imagem corporal em relação ao estado nutricional, adiposidade corporal e sintomas de anorexia e bulimia. No modelo simples, foi verificada associação da insatisfação com a imagem corporal com IMC e \%G. Quando o modelo foi ajustado para todas as variáveis, a insatisfação manteve-se associada somente ao IMC, sendo que as adolescentes com excesso de peso apresentaram 2,64 vezes mais chance de estarem insatisfeitas com a imagem corporal em relação àquelas com IMC normal.

Tabela 2 - OR para insatisfação com a imagem corporal (categoria de referência: satisfeita) em adolescentes do sexo feminino segundo indicadores antropométricos e presença de sintomas de anorexia e bulimia - Santa Maria, RS Brasil, 2007.

\begin{tabular}{llccc}
\hline Variáveis & OR & IC95\% & OR* & IC95\% \\
\hline IMC & 1,00 & & 1,00 & $0,02-1,54$ \\
$\quad$ Normal & 0,19 & $0,03-1,46$ & 0,19 & $1,02-6,83$ \\
$\quad$ Baixo peso & 3,07 & $1,63-5,79$ & 2,64 & \\
$\quad$ Excesso de peso & & & $0,55-2,56$ \\
\% & 1,00 & $0,81-3,22$ & 1,00 & $0,42-3,82$ \\
$\quad$ Normal & 1,61 & $1,49-6,34$ & 1,27 & \\
$\quad$ Alto & 3,08 & & 1,00 & $0,90-3,30$ \\
$\quad$ Muito alto & 1,00 & $0,89-3,03$ & 1,72 & \\
EAT & 1,64 & & \\
$\quad$ Negativo & Positivo & & & \\
\hline
\end{tabular}

$\% \mathrm{G}=$ percentual de gordura; EAT = teste de atitudes alimentares; IC95\% = intervalo de confiança de 95\%; IMC = índice de massa corporal; OR = razão de chances (odds ratio).

* OR ajustada para todas as variáveis.

\section{Discussão}

A prevalência de insatisfação com a imagem corporal encontrada nas adolescentes do presente estudo (25,3\%) é maior que a observada nos estudos nacionais que utilizaram o mesmo instrumento ${ }^{6,11}$. Alves et al. ${ }^{6}$ encontraram prevalência de $18,8 \%$ de insatisfação em escolares do sexo feminino (10 a 19 anos) do município de Florianópolis (SC). No estudo de Branco et al. ${ }^{11}$, a insatisfação corporal esteve presente em 18,5\% das estudantes de São Paulo (SP) (14 a 19 anos). Os resultados encontrados nas adolescentes de Santa Maria são similares aos achados em adolescentes do sexo feminino da Espanha $(22,1 \%)^{28}$.

Além disso, esses resultados são semelhantes aos observados em estudantes universitárias. Das alunas do curso de Nutrição da Universidade Federal do Rio de Janeiro (UFRJ), Rio de Janeiro (RJ), 18,6\% mostraram-se insatisfeitas com a sua imagem corporal ${ }^{12}$. Em alunas da Universidade Metodista de Piracicaba (SP), encontraram-se prevalências de 17,3 e 22,2\% nos cursos de Nutrição e Psicologia, respectivamente ${ }^{29}$.

Em relação aos distúrbios alimentares, resultados preocupantes foram encontrados nas adolescentes do presente estudo, pois a prevalência de escolares que apresentaram sintomas de anorexia e bulimia $(27,6 \%)$ foi superior à encontrada em outros estudos nacionais ${ }^{6,20,21,30}$ e internacionais ${ }^{3,28,31,32}$, o que demonstra que grande parte das escolares analisadas possui importantes fatores de risco para o desenvolvimento desses distúrbios.

Um estudo realizado com estudantes de escolas públicas (ambos os sexos) de cinco cidades do interior de Minas Gerais revelou que $15,8 \%$ das adolescentes do sexo feminino ( 7 a 19 anos) apresentavam comportamentos alimentares anor- mais $^{20}$. Em alunas de 15 a 18 anos de uma escola particular da cidade de São Paulo, a prevalência de sintomas de anorexia e bulimia foi de $21,1 \%{ }^{30}$. No Canadá, $14 \%$ das escolares (12 a 18 anos) demonstraram risco de desenvolvimento de anorexia e bulimia ${ }^{3}$. Nesses estudos, foi utilizado o ponto de corte de 20 pontos para o EAT-26. Outras pesquisas que optaram pelo escore 21 nesse questionário identificaram prevalências de $8,3^{21}, 15^{31}$ e $15,6 \%{ }^{6}$ de sintomas de anorexia e bulimia em adolescentes do sexo feminino. Em estudo realizado na Espanha, ao utilizar o EAT-40, questionário que originou o EAT-26, foi verificado que $16,3 \%$ das moças apresentavam comportamentos alimentares anormais ${ }^{28}$.

Dessa forma, parece que as estudantes de escolas públicas da cidade de Santa Maria estão mais propensas a desenvolver anorexia e bulimia em relação às adolescentes de outras regiões brasileiras ${ }^{6,20,21,30}$ e também de outros países como o Canadá ${ }^{3}$ e EUA ${ }^{31}$.

A literatura tem revelado que a presença de distúrbios alimentares é bastante evidente na rede pública de ensino ${ }^{6,11,32}$, corroborando os resultados do presente estudo.

A insatisfação com a imagem corporal não apresentou associação com os sintomas de anorexia e bulimia, o que não era esperado neste estudo. Alves et al. ${ }^{6}$ encontraram forte associação entre essas duas variáveis em adolescentes de Florianópolis. Uma possível explicação para essa ocorrência pode estar relacionada ao fato de que a insatisfação com a imagem corporal está presente nas adolescentes independentemente de apresentarem ou não comportamentos de risco para o desenvolvimento de distúrbios alimentares.

Neste estudo, observou-se associação entre insatisfação com a imagem corporal e o estado nutricional, sendo que as adolescentes com excesso de peso apresentaram maior insa- 
tisfação com a imagem corporal em relação àquelas eutróficas. Esses resultados corroboram os achados de Branco et al. ${ }^{11}$, que, ao analisar adolescentes (14 a 19 anos) de ambos os sexos, observaram, no sexo feminino, maior insatisfação naquelas com sobrepeso e obesidade. Entretanto, Bosi et al. ${ }^{12}$ não encontraram essa mesma relação em estudantes universitárias, pois entre aquelas insatisfeitas, $82,9 \%$ eram eutróficas e apenas $11,4 \%$ apresentavam excesso de peso.

Até o presente momento, ainda não tinha sido reportada na literatura a relação entre percentual de gordura e insatisfação com a imagem corporal. Neste estudo, apesar de ter sido observada associação entre essas duas variáveis no teste qui-quadrado, na análise de regressão essa associação não foi evidenciada. Nesse contexto, observa-se um número crescente de adolescentes que, mesmo apresentando uma quantidade de gordura corporal adequada para a idade, estão insatisfeitas com a imagem corporal, pois almejam ser cada vez mais magras. Esses resultados refletem a magnitude da influência da mídia e da sociedade em geral sobre a imagem corporal dessas escolares, ao determinar um padrão de beleza excessivamente magro.

A principal limitação do estudo refere-se ao seu delineamento de corte transversal, o que dificulta verificar a relação causa-efeito entre as variáveis. Por outro lado, ressalta-se que este é o primeiro estudo a investigar a prevalência de insatisfação com a imagem corporal e sua associação com indicadores antropométricos e distúrbios alimentares em adolescentes da cidade de Santa Maria.

Os resultados encontrados no presente estudo permitem concluir que as prevalências de insatisfação com a imagem corporal e de sintomas de anorexia e bulimia foram elevadas, demonstrando a necessidade de intervenções nas escolas públicas com o objetivo de prevenir o desenvolvimento de distúrbios alimentares em adolescentes do sexo feminino. Os sintomas de anorexia e bulimia e o \%G não apresentaram associação com a insatisfação com a imagem corporal. Já o estado nutricional apresentou tal associação, mostrando que o excesso de peso prediz a maior insatisfação com a imagem corporal em adolescentes do sexo feminino.

\section{Referências}

1. Slade PD. Body Image in anorexia nervosa. Br J Psychiatry Suppl. 1988;(2):20-2.

2. Campagna VN, Souza AS. Corpo e imagem corporal no início da adolescência feminina. Bol psicol. 2006;56(124):9-35.

3. Jones JM, Bennett S, Olmsted MP, Lawson ML, Rodin G. Disordered eating attitudes and behaviors in teenaged girls: a school-based study. CMAJ. 2001;165(5):547-52.

4. Tiggemann M, Pickering AS. Role of television in adolescent women's body dissatisfaction and drive for thinness. Int J Eat Disord. 1996;20(2):199-203.

5. Nunes MA, Olinto MT, Barros FC, Camey S. Influência da percepção do peso e do índice de massa corporal nos comportamentos alimentares anormais. Rev Bras Psiquiatr. 2001;23(1):21-7.
6. Alves E, Vasconcelos FA, Calvo MC, Neves J. Prevalência de sintomas de anorexia nervosa e insatisfação com a imagem corporal em adolescentes do sexo feminino do município de Florianópolis, Santa Catarina, Brasil. Cad Saude Publica. 2008;24(3):503-12.

7. Morandé G, Celada J, Casas JJ. Prevalence of eating disorders in a Spanish school-age population. J Adolesc Health. 1999;24(3):212-9.

8. Nunes MA, Bagatini LF, Abuchaim AL, Kunz A, Ramos D, Silva JA, et al. Distúrbios da conduta alimentar: considerações sobre o Teste de Atitudes Alimentares (EAT). Rev ABP-APAL. 1994;16(1):7-10.

9. Borges NJ, Sicchieri JM, Ribeiro RP, Marchini JS, dos Santos JE. Transtornos alimentares - quadro clínico. Medicina (Ribeirão Preto). 2006;39(3):340-8.

10. Practice guideline for the treatment of pacients with eating disorders (revision). American Psychiatric Association Work Group on Eating Disorders. Am J Psychiatry. 2000;157(1 Suppl):1-39.

11. Branco LM, Hilário MO, Cintra IP. Percepção e satisfação corporal em adolescentes e a relação com seu estado nutricional. Rev Psiquiatr Clin. 2006;33(6):292-6.

12. Bosi ML, Luiz RR, Morgado CM, Costa ML, de Carvalho RJ. Autopercepção da imagem corporal entre estudantes de nutrição: um estudo no município do Rio de Janeiro. J Bras Psiquiatr. 2006;55(2):108-13.

13. Sistema de Estatísticas Educacionais. [website] Brasil, Ministério da Educação. Disponível em: <www.edudatabrasil.inep.gov.br>. Acessado em 15/04/2007.

14. Fonseca JS, Martins GA. Curso de Estatística. 6 ed. São Paulo: Atlas; 1996.

15. Cordás TA, Castilho S. Imagem corporal nos transtornos alimentares - Instrumento de Avaliação: "Body Shape Questionnaire". Psiquiatr Biol. 1994;2(1):17-21.

16. Cooper PJ, Taylor MJ, Cooper Z, Fairbun CG. The development and validation of the body shape questionnaire. Int J Eat Disord. 1987;6(4):485-94.

17. Manetta MC. Validade interna, dimensionalidade e desempenho da escala BSQ "Body Shape Questionaire" em uma população de estudantes universitários [dissertação]. São Paulo: Universidade Federal de São Paulo; 2002.

18. Garner DM, Olmsted MP, Bohr Y, Garfinkel PE. The eating attitudes test: psychometric features and clinical correlates. Psychol Med. 1982;12(4):871-8.

19. Bighetti F. Tradução e validação do Eating Attitudes Test (EAT-26) em adolescentes do sexo feminino na cidade de Ribeirão Preto - SP [dissertação]. Ribeirão Preto: Universidade de São Paulo; 2003.

20. Vilela JE, Lamounier JA, Dellaretti Filho MA, Barros Neto JR, Horta GM. Transtornos alimentares em escolares. J Pediatr (Rio J). 2004;80(1):49-54.

21. Chiodini JS, Oliveira MR. Comportamento alimentar de Adolescentes: aplicação do EAT-26 em uma escola pública. Saude Rev. 2003;5(9):53-8.

22. Alvarez BR, Pavan AL. Alturas e comprimentos. In: Petroski EL, ed. Antropometria: técnicas e padronizações. $3^{\mathrm{a}}$ ed. Blumenau: Nova Letra; 2007. p. 31-44.

23. Cole TJ, Flegal KM, Nicholls D, Jackson AA. Body mass index cut offs to define thinness in children and adolescents: international survey. BMJ. 2007;335:194.

24. Cole TJ, Bellizzi MC, Flegal KM, Dietz WH. Establishing a standard definition for child overweight and obesity worldwide: international survey. BMJ. 2000;320(7244):1240-3.

25. Benedetti TR, Pinho RA, Ramos VM. Dobras cutâneas. In: Petroski EL, ed. Antropometria: técnicas e padronizações. $3^{\mathrm{a}}$ ed. Blumenau: Nova Letra; 2007. p. 45-56.

26. Slaughter MH, Lohman TG, Boileau RA, Horswill CA, Stillman RJ, Van Loan $\mathrm{MD}$, Bemben DA. Skinfold equations for estimation of body fatness in children and youth. Hum Biol. 1988;60(5):709-23.

27. Lohman TG. The use of skinfolds to estimate body fatness on children and youth. J Phisical Educ Recr Dance. 1987;58:98-103.

28. Ballester Ferrando De Gracia Blanco M, Patiño Masó J, Suñol Gurnés C, Ferre Avellí M. [Eating attitudes and body satisfaction in adolescents: a prevalence study.] Actas Esp Psiquiatr. 2002;30(4):207-12.

29. Stipp LM, Oliveira MR. Imagem corporal e atitudes alimentares: diferenças entre estudantes de nutrição e de psicologia. Saude Rev. 2003;5(9):47-51.

30. Dunker KL, Philippi ST. Hábitos e comportamentos alimentares de adolescentes com sintomas de anorexia nervosa. Rev Nutr. 2003;16(1):51-60.

31. Pastore DR, Fisher M, Friedman SB. Abnormalities in weight status, eating attitudes, and eating behaviors among urban high school students: correlations with self-esteem and anxiety. J Adolesc Health. 1996;18(5):312-9.

32. Rodriguéz A, Novalbos JP, Martínez JM, Ruiz MA, Fernández JR, Jiménez D Eating Disorders and altered eating behaviors in adolescents of normal weight in a Spanish city. J Adolesc Health. 2001;28(3):338-45. 